\title{
Esforços de Inovação de Pequenos Negócios na América Latina
}

\author{
Christian Daniel Falaster ${ }^{\mathrm{A}}$ (D) e Priscila Rezende da $\operatorname{Costa}^{\mathrm{B}}$ (iD) \\ ${ }^{\text {A }}$ Programa de Pós-graduação em Administração, Universidade Regional de Blumenau - FURB, Blumenau, SC, Brasil \\ ${ }^{B}$ Programa de Pós-graduação em Administração, Universidade Nove de Julho - UNINOVE, São Paulo, SP, Brasil
}

\section{Detalhes Editoriais}

Sistema double-blind review

Histórico do Artigo

Recebido: 01 de Jul., 2020

Revisado: 05 de Out., 2020

Aceito: 25 de Nov., 2020

Disponível online: 25 de Dez, 2020

CLASSIFICAÇÃo JEL: 032

ARTIGO ID: 1957

Editor Chefe

Dr. Dennys Eduardo Rossetto (D)

SKEMA Business School

Editor Científico Responsável

Dra. Vânia Nassif (D)

Universidade 9 de Julho, UNINOVE

Revisão Ortográfica e Gramatical Dra. Mônica Império Costa (iD Palavra Seleta Revisão Textual

Financiamento:

FURB: $672 / 2019$

CNPq: $422922 / 2018-8$

309294/2019-3

Cite como:

Falaster, C. D., e Costa, P, R. (2021). Esforços de Inovação de Pequenos Negócios na América Latina. Revista de Empreendedorismo e Gestão de Pequenas Empresas 10(1), Artigo e1957. https://doi.org/10.14211/ regepe.v10i1.1957

Contato dos autores:

Christian Daniel Falaster christianfalaster@gmail.com

Priscila Rezende da Costa priscilarezende@yahoo.com.br

\section{Resumo}

Objetivo do estudo: Analisar os efeitos das dinâmicas institucionais nos esforços de inovação de pequenos negócios na América Latina. Metodologia/abordagem: Utilizamos uma base de dados do Banco Mundial com 11.446 pequenos negócios latino-americanos, que responderam questões relativas aos seus esforços de inovação (de produto, de processo e investimentos em pesquisa e desenvolvimento) nos últimos três anos. Para avaliar o efeito das dinâmicas institucionais (reformas e reversões), foram captados dados do Índice de Liberdade Econômica. Principais resultados: Concluímos que, nos pequenos negócios de países latino-americanos, os esforços de inovação podem estar mais ligados à preparação para o enfrentamento de um ambiente institucionalmente ineficiente do que ao aproveitamento dos ambientes que tiveram uma melhora institucional. Contribuições teóricas/metodológicas: Os movimentos desses pequenos negócios possivelmente se relacionam à busca por sobrevivência em um ambiente incerto, contrariando, assim, a suposição de que haveria mais esforços de inovação quando o ambiente fosse propício a ela. Relevância/originalidade: Este estudo é valioso por possibilitar a análise das dinâmicas institucionais, que podem levar a determinados tipos de resposta nos esforços de inovação de pequenos negócios. Contribuições sociais/para a gestão: este estudo também demonstra se a formulação de reformas institucionais pode impactar os pequenos negócios, no contexto latino-americano, sendo relevante, desse modo, para o desenvolvimento de políticas públicas.

Palavras-chave: Esforços de inovação; Dinâmicas institucionais; Reformas institucionais; Reversões institucionais.

\section{(c) 2021 ANEGEPE Ltda. Todos os direitos reservados.}

\begin{abstract}
Purpose of the study: in this paper, we analyze the effects of institutional dynamics on innovation efforts of small businesses in Latin America. Methodology/approach: we use a survey sample of 11,446 small Latin American businesses from The World Bank; The survey comprised answered questions regarding their innovation efforts in the last three years: product innovation, process innovation and investments in research and development; To assess the effect of institutional dynamics (reforms and reversals), data from the Economic Freedom Index were captured. Main results: we conclude that in small businesses in Latin American countries, innovation efforts may be more linked to the preparation to face an institutionally inefficient environment than to take advantage of the environments that have had an institutional improvement. Theoretical/methodological contributions: these small business movements may be more linked to the search for survival in an uncertain environment, contradicting the expected effects that there would be more innovation efforts when the environment was conducive to it. Relevance/originality: this study is valuable because it allows the analysis of what types of institutional dynamics can lead to what types of responses in innovation efforts of small businesses. Social/management contributions: the study also contributes by demonstrating whether the formulation of institutional reforms can impact small businesses in the Latin American context, thus being important for the development of public policies.
\end{abstract}

Keywords: Innovation efforts; Institutional dynamics; Institutional reforms; Institutional reversals 


\section{INTRODUÇÃO}

Esforços para o desenvolvimento da inovação são importantes à competitividade empresarial (Freel, 2005), pois constituem uma capacidade altamente sensível ao contexto no qual as organizações se inserem (Solleiro e Castañon, 2005). Sendo assim, empresas instaladas fora do eixo dos países desenvolvidos tendem a realizar relativamente pouca inovação em seus negócios (Szogs, 2008); e, ainda assim, elas precisam inovar para se manterem competitivas em relação às ameaças externas e aos seus concorrentes internos. Nesse sentido, destacam-se as iniciativas de alguns países latino-americanos, que têm implementado reformas institucionais e econômicas, com o objetivo de tornar suas empresas mais inovadoras e competitivas (Dau, 2012, 2013).

A literatura traz fatores empresariais e econômicos que determinam os esforços de inovação das empresas, como: a estrutura do mercado, a apropriabilidade da demanda, a abertura internacional e a colaboração tecnológica (Kannebley Jr. et al., 2005; Kafouros et al., 2015; Papazoglou e Spanos, 2018).

Fatores institucionais, como: a qualidade regulamentar, o controle da corrupção, a governança e a transparência institucional (Sirmon et al., 2007; Kaufmann et al., 2011; Barasa et al., 2017; Wang, 2018) também são de grande importância para as empresas, em um ambiente concorrencial, visto que reduzem as incertezas relacionadas aos negócios (North, 1991).

Por outro lado, reformas institucionais, especificamente as pró-mercado, têm despontado em alguns países, em especial na América Latina, com o objetivo de melhorar a eficiência das empresas e o desempenho econômico (Dau, 2012, 2013; Banalieva et al., 2018).

O papel do avanço institucional nos esforços de inovação das empresas é conhecido e está diretamente relacionado à qualidade do ambiente institucional. Enquanto sua existência pode estimular comportamentos inovadores, reduzindo as incertezas e facilitando a coordenação entre os agentes econômicos (Acemoglu e Robinson, 2008; Alonso e Garcimartín, 2013); o baixo nível dessa qualidade, em contrapartida, leva a comportamentos improdutivos, por vezes oportunistas, e amplia as incertezas, reduzindo a propensão de inovar nas firmas (Greif, 2006; Alence, 2004).

Nos países menos desenvolvidos, o avanço institucional ainda é um desafio, embora haja uma tentativa de desenvolvimento pela liberdade econômica, por meio de reformas estruturais pró-mercado (Cuervo-Cazurra and Dau, 2009). Os países latinoamericanos, por exemplo, têm características importantes em seus contextos, tais como a restrição de recursos humanos e tecnológicos qualificados, quando comparados a países desenvolvidos, resultado de um fator histórico de economia de industrialização tardia (Trevino et al., 2008).

Além da complexidade intrínseca às dinâmicas institucionais dos países latino-americanos, vale destacar que os desafios impostos pelas restrições de recursos são ainda maiores para pequenos negócios, se colocados em paralelo aos negócios de maior porte (Tolstoy, 2009). Consequentemente, os pequenos negócios devem buscar alternativas inovadoras, a fim de reconfigurar seus ativos para lidar com a dinâmica do ambiente institucional (Rosenberg, 2010).

A escassez de recursos torna a inovação custosa, levando as empresas latino-americanas a decidir entre alocar recursos para potencializar os esforços de inovação ou reforçar sua posição nos produtos e em processos que já possuem. Tal decisão pode demandar investimento em pesquisa e desenvolvimento (P\&D) e a introdução de novos produtos e processos (OECD, 2005; Goedhuys et al., 2013).

Neste artigo, analisamos se essa decisão é também influenciada por fatores institucionais, como as reformas e as reversões, pois elas estão relacionadas aos níveis de qualidade institucional percebidos pelos gestores (Kaufmann et al., 2011; Barasa et al., 2017; Wang, 2018).

Sendo assim, partindo do pressuposto que as reformas institucionais seja, possivelmente, promotoras de efeitos positivos nos esforços de inovação dos pequenos negócios, enquanto as reversões institucionais, por exigirem ações mais conservadoras, resultam em um menor esforço, esta investigação quantitativo-explicativa, que remete ao nível da firma, tem o objetivo de examinar a influência das dinâmicas institucionais nos esforços de inovação dos pequenos negócios. Foram analisados, para tanto, dados de 11.446 mil pequenos negócios de países latino-americanos, particularmente os que responderam a questões relativas aos esforços de inovação do Enterprise Survey do Banco Mundial (World Bank, 2017).

Os dados secundários, considerados na pesquisa, referemse a 2006 e 2017, e foram organizados da seguinte maneira: (a) para a variável independente "dinâmicas institucionais", dividida em "reformas e reversões institucionais", foi adotado o grau de mudança do Economic Freedom Index (Banalieva et al., 2018); e (b) para a variável dependente "esforços de inovação", foram selecionados os indicadores de "investimento em P\&D", de "introdução de novos produtos" e de "introdução de novos processos" do Enterprise Survey do Banco Mundial (World Bank, 2017), seguindo os padrões do Manual de Oslo (OECD, 2005).

Vale destacar que são pontuais as pesquisas empíricas acerca da relação entre as dinâmicas institucionais e os esforços de inovação das empresas nos países que buscam o desenvolvimento, como os latino-americanos. Isso pode estar associado ao fato de que é recente nesses países a disponibilização sistematizada de dados sobre o esforço de inovação das empresas, decorrente da tardia estruturação dos sistemas nacionais de inovação (Ayyagari et al., 2012; Goedhuys e Veugelers, 2012, Goedhuys et al., 2013).

Sobre as contribuições, nós enfatizamos que este estudo: (a) é o primeiro a avaliar conjunta e empiricamente fatores econômicos de dinâmicas institucionais (reformas e reversões), com a finalidade de explicar os esforços de inovação dos pequenos negócios (alocação de recursos), considerando como contexto de análise um número alargado de firmas de países latino-americanos; (b) possibilita a análise dos tipos de dinâmicas institucionais e de suas respectivas respostas em esforços de inovação dos pequenos negócios; e (c) demonstra como a formulação de reformas institucionais pode impactar os pequenos negócios, no contexto latino-americano, sendo assim importante para o desenvolvimento de políticas públicas.

\section{REFERENCIAL TEÓRICO}

Os ativos tangíveis e intangíveis constituem o fundamento do desempenho empresarial distintivo (Ireland et al., 2003; Barney e Arikan, 2001) e integram a base dos recursos de uma empresa (Mahoney, 1995; Sirmon et al., 2007); por isso, para se obter valor, eles precisam ser adequadamente gerenciados.

O investimento em P\&D é um exemplo de recurso no nível da firma, que impulsiona o esforço de inovação (Goedhuys, 2007; Goedhuys e Sleuwaegen, 2010). Se gerenciado adequadamente, ele amplia as possibilidades de se gerar valor, tendo-se, como 
consequência, a introdução de novos produtos e processos no mercado (Crespi e Zuniga, 2012; Bradley et al., 2012).

Embora a relação entre investimento em P\&D, introdução de novos produtos e processos, e inovação seja frequentemente considerada positiva e benéfica à firma (Arundel et al., 2007), nota-se, nos países em desenvolvimento, que tais relações guardam variações (Crespi e Zuniga, 2012). Na Ásia, por exemplo, estudos apontam uma associação positiva (Lee e Kang, 2007; Wang e Lin, 2012; ao passo que evidências no Chile e no México não corroboram esse resultado (Wang e Lin, 2012). Nos países da África, por sua, vez, conforme Goedhuys (2007), observouse uma relação positiva entre $P \& D$ e inovação de produto na Tanzânia; sendo que ela foi especialmente considerada um componente primordial à estratégia de inovação para fabricantes de roupas e empresas têxteis do Quênia, de Kamau e Munandi (2009).

Além da gestão interna dos recursos, as empresas de países em desenvolvimento são continuamente desafiadas a lidar com as dinâmicas institucionais, muitas vezes caracterizadas pelo alto grau de instabilidade política, de corrupção generalizada e por uma fraca proteção dos direitos de propriedade (Bräutigam e Knack, 2004)). Na maioria desses países, ainda vigora a má governança, decorrente da presença de instituições ineficientes (Abed e Gupta, 2002), o que explica as taxas de crescimento irregulares em tais localidades (Olson et al., 2000). Estudos empíricos, como o de Glaeser et al. (2004) e de Acemoglu e Robinson (2008), ratificam o papel crítico das instituições, no que tange ao crescimento econômico dos países em desenvolvimento.

Sendo assim, neste artigo, nosso posicionamento teórico está alinhado à nova economia institucional (NEI), seguindo trabalhos como os de Williamson (2000), North (1990, Freel, 2005, e Henisz (2000). Embora notáveis estudos na área de inovação já tenham utilizado essa perspectiva nos tópicos relativos a sistemas nacionais de inovação (Nelson e Nelson, 2002), à relação entre instabilidade política e inovação (Bhattacharya et al., 2017), e à complexidade institucional (Wu e Park, 2019), entre outros, a dinâmica das instituições dos países e os seus efeitos sobre a inovação continuam sendo temas que pedem um maior aprofundamento investigativo.

Como o ambiente institucional dos países não é estático, desenvolvendo-se de acordo com mudanças institucionais (North, 1991) que, na maioria das vezes, são graduais e geradas ao longo de décadas, a partir da definição de leis e de políticas públicas (Williamson, 2000), a principal função das instituições é restringir possíveis ações dos decisores e estabelecer padrões de comportamento condizentes às regras formais e informais do ambiente (North, 1990).

Um ambiente institucional adequado ao desenvolvimento das empresas geralmente apresenta a previsibilidade das instituições e da mudança institucional, sendo as regras claras e as ações arbitrárias ou oportunistas coibidas (Henisz, 2000). Com o comportamento das outras partes previsível, em uma transação, é possível reduzir a incerteza nessas instituições (North, 1991).

As dinâmicas institucionais são importantes para os ambientes, pois mudam as "regras do jogo" neles encontradas. Por isso, reformas institucionais pró-mercado têm sido frequentes em diferentes partes do globo, com fins de, principalmente, melhorar seus ambientes de negócios, tornar suas empresas mais competitivas e resolver imperfeições mercadológicas originadas por políticas protecionistas e centralizadoras (Banalieva et al., 2018).
Nesse sentido, nas últimas décadas, os países latino-americanos desenvolveram uma série de reformas, que abarcam não apenas as leis e as regras para a competição nos negócios, mas também modificações fiscais, políticas, educacionais e contra a corrupção (Trevino et al., 2008), permitindo-lhes descentralizar e liberalizar suas economias para o trânsito de mercado (Dau, 2013).

As mudanças institucionais pró-mercado tendem a: (a) diminuir a intervenção do governo nos negócios e na economia (Cuervo-Cazurra and Dau, 2009); (b) melhorar a liberdade econômica dos países (Banalieva et al., 2018); e (c) aumentar a rentabilidade das empresas, assim como a atratividade de país onde se localizam para o investimento direto externo (Dau, 2013).

Vale destacar, todavia, que nem sempre as mudanças institucionais são pró-mercado. Por exemplo, governos, muitas vezes, geram mudanças caracterizadas como reversões institucionais, ou seja, são retrocessos em que uma economia mais adequada passa a se tornar inóspita a algumas empresas (Banalieva et al., 2018), restringindo, com isso, sua liberdade e resultando em menos oportunidades (Dau and Cuervo-Cazurra, 2014).

Mudanças de perspectivas políticas do governo, especialmente no executivo, podem acarretar novas ou diferentes políticas e dinâmicas institucionais. Essas mudanças, de acordo com Banalieva et al., 2018, são de quatro tipos: (1) reformas crescentes - quando uma melhora institucional é seguida por outra ainda maior no ano seguinte; (2) reformas decrescentes - quando uma melhora institucional é seguida por outra, no ano subsequente, relativamente menor do que a primeira; (3) reversões crescentes - quando uma deterioração institucional é seguida por outra ainda maior, no ano subsequente; e, finalmente, (4) reversões decrescentes - quando uma deterioração institucional é seguida por outra menor, no ano subsequente.

Quando as reformas institucionais pró-mercado acontecem de forma crescente, as empresas percebem a qualidade institucional do país, que melhora com maior intensidade. Dessa forma, a incerteza sobre as políticas governamentais diminui (Banalieva et al., 2018), assim como a perspectiva de um ambiente institucional melhor para os negócios aumenta (Dau, 2012). Espera-se que, após reformas crescentes, as empresas intensifiquem seus esforços de inovação, haja vista as perspectivas de respeito maior aos direitos de propriedade e de condições de mercado mais eficientes, que favorecem quem realiza esforços de inovação. Sendo assim, apresentamos a:

Hipótese 1: reformas crescentes estão diretamente relacionadas aos esforços de inovação.

Por outro lado, quando as reformas institucionais prómercado são decrescentes, elas mostram uma perspectiva negativa e incerta sobre o futuro (Banalieva et al., 2018). Assim, quando há uma redução nas reformas pró-mercado, espera-se que as empresas percebam o ambiente como incerto para o futuro e adotem uma tendência de redução em seus esforços de inovação, recorrendo a estratégias mais conservadoras e mantendo suas atividades mais próximas daquilo que consideram saber fazer bem. Nesse sentido, propomos a:

\section{Hipótese 2: reformas decrescentes estão inversamente relacionadas aos esforços de inovação.}

Quando há reversões no ambiente institucional, que o fazem sofrer uma deterioração em relação ao passado, o futuro tende a ser percebido como mais complicado para a competição franca entre as empresas (Dau, 2013). Tais reversões podem acontecer 
de duas formas distintas (Banalieva et al., 2018): (1) reversões crescentes, que resultam em uma perspectiva de condições institucionais cada vez piores, em função de ambientes menos livres para os negócios; e (2) reversões decrescentes, que podem significar uma mudança possivelmente positiva, no longo prazo. Nesse sentido, espera-se que, face a reversões crescentes, as empresas recorreram a estratégias mais conservadoras, pois vislumbram um ambiente inseguro e incerto no futuro, diminuindo, com isso, seus esforços de inovação. Assim, temos a:

Hipótese 3: reversões crescentes estão inversamente ligadas aos esforços de inovação.

Ao contrário das reversões crescentes, as decrescentes representamumapossívelmudançanaspolíticasgovernamentais, diminuindo o deterioramento das instituições e, possivelmente, levando a um avanço institucional, em médio ou longo prazo. Dessa forma, esforços de inovação serão mais comuns quando as empresas perceberem reversões decrescentes, pois elas podem sinalizar futuras melhoras e ambientes mais adequados aos negócios. Sendo assim, propomos a:

Hipótese 4: reversões decrescentes estão diretamente ligadas aos esforços de inovação.

\section{MÉTODO}

Para esta investigação, utilizamos dados quantitativos de 11.446 mil pequenos negócios de países latino-americanos, a partir dos microdados da Enterprise Survey do Banco Mundial (World Bank, 2017), que é uma pesquisa realizada com organizações de todos os tamanhos e setores, com o objetivo de analisar o ambiente de negócios naquele país.

Vale destacar que $74 \%$ da amostra é representada por empresas que empregam até 99 trabalhadores, sendo, portanto, constituída, em sua maioria, por pequenos negócios. Além disso, a amostra final levou em consideração somente as organizações que responderam a questões relativas aos seus esforços de inovação nos últimos três anos, indicando se realizaram ou não inovações de produto, de processo ou investimentos em P\&D.

Estudos quantitativos explicativos são adequados para testar hipóteses, uma vez que oferecem informações resumidas sobre várias características; e as técnicas de coleta e de análise de dados são estruturadas e objetivas. Ademais, por haver a preocupação com a representatividade, amostras grandes e complexas, em geral, são adotadas (Hair et al., 2005).

Para testar as hipóteses, adotamos o método de regressão logística (Hair et al., 2005) e os padrões da Enterprise Survey, que estão de acordo o Manual de Oslo (OECD, 2005).

Para as variáveis dependentes deste estudo, que refletem os esforços de inovação das empresas, foram utilizadas as respostas das empresas às seguintes perguntas: "Essa empresa introduziu um novo produto no último ano?", "Essa empresa introduziu um novo processo no último ano?" e "Essa empresa investiu em pesquisa e desenvolvimento no último ano?".

Para as variáveis independentes, utilizamos a classificação de Banalieva et al., 2018 sobre reformas institucionais pró-mercado, divididas em reformas crescentes, reformas decrescentes, reversões crescentes e reversões decrescentes. Para tanto, o ano fiscal, foco da pesquisa, recebeu a denominação de t; o ano anterior, de t-1; e o ano antecessor a este, de t-2.

Foram, então, calculadas as variações no Economic Freedom Index (EFI) (Índice de Liberdade Econômica), da Heritage Foundation (2019), entre $t-2$ e $t-1$, e entre $t-1$ e $t$, nomeados V1 e V2, seguindo as fórmulas (V1 = (EFIt-1 - EFIt-2)EFI/t-2) e (V2 $=($ EFIt - EFIt-1)/EFIt-1). V1 e V2 positivos indicavam reformas institucionais pró-mercado; ao passo que V1 e V2 negativos apontavam para reversões. As reformas crescentes foram classificadas como " 1 ", quando $\mathrm{V} 1<\mathrm{V} 2$; e como " 0 ", em outros casos; e as decrescentes, por sua vez, como "1", quando V1>V2; e como " 0 ", em outros casos. As reversões crescentes receberam a classificação "1", quando V1<V2; e "0", em outros casos; e as decrescentes, "1", quando V1>V2; e "0", em outros casos.

Como variáveis de controle, foram utilizados: (1) o nível país, a partir de dados do Banco Mundial (2019), considerando a variação do PIB e da inflação (para controle do momento econômico do país); e a variação do PIB per capita (para controle das características econômicas do país); (2) o nível empresa, por meio de dados da Enterprise Survey, como a idade da empresa (para controle da experiência no mercado), a origem do capital (para controle das possíveis variações de propriedade), e o número de empregados (para controle do tamanho da empresa);

\begin{tabular}{|c|c|c|c|c|}
\hline Variáveis & Descrição & Indicadores & Transformações & Fontes \\
\hline \multirow[t]{3}{*}{ Dependentes } & \multirow[t]{3}{*}{ Esforços de inovação } & Investimento em P\&D nos últimos três anos. & \multirow{3}{*}{$\begin{array}{l}1=\text { sim } \\
0=\text { não }\end{array}$} & \multirow{3}{*}{$\begin{array}{c}\text { Enterprise Survey } \\
\text { do Banco Mundial } \\
\text { (World Bank, 2017) }\end{array}$} \\
\hline & & $\begin{array}{l}\text { Introdução de novos produtos nos últimos } \\
\text { três anos. }\end{array}$ & & \\
\hline & & $\begin{array}{l}\text { Introdução de novos processos nos últimos } \\
\text { três anos. }\end{array}$ & & \\
\hline Independentes & $\begin{array}{r}\text { Reformas e reversões } \\
\text { institucionais }\end{array}$ & $\begin{array}{l}\text { Reformas crescentes e decrescentes } \\
\text { Reversões crescentes e decrescentes }\end{array}$ & $\begin{array}{l}1=\operatorname{sim} \\
0=\text { não }\end{array}$ & $\begin{array}{l}\text { Economic Freedom Index } \\
\text { (Banalieva et al., 2018) }\end{array}$ \\
\hline \multirow[t]{8}{*}{ Controles } & \multirow[t]{3}{*}{ Por país } & Variação do PIB & \% de variação do PIB, em relação ao ano anterior. & \multirow[t]{3}{*}{ World Bank (2017) } \\
\hline & & PIB per capita & Em milhões de dólares, constantes de 2010. & \\
\hline & & Inflação & $\begin{array}{l}\text { \% de variação nos preços gerais, } \\
\text { em relação ao ano anterior. }\end{array}$ & \\
\hline & \multirow[t]{4}{*}{ Por empresa } & Idade da empresa & Número de anos desde a fundação. & \multirow{5}{*}{$\begin{array}{l}\text { Enterprise Surveys } \\
\text { (World Bank, 2017) }\end{array}$} \\
\hline & & Posse estrangeira & $\begin{array}{l}\text { \% de participação acionária estrangeira } \\
\text { nas ações da empresa. }\end{array}$ & \\
\hline & & Posse governamental & $\begin{array}{l}\text { \% de participação acionária governamental } \\
\text { nas ações da empresa. }\end{array}$ & \\
\hline & & Número de empregados & Número de empregados na empresa. & \\
\hline & Por setor & $\begin{array}{l}\text { Dicotômica para cada setor, utilizando SIC } \\
\text { Code de dois dígitos. }\end{array}$ & $\begin{array}{l}1=\operatorname{sim} \\
0=\text { não }\end{array}$ & \\
\hline
\end{tabular}

Tab. 01

Síntese agregada das variáveis

Fonte: Elaborada pelos autores 
e (3) o nível setor, com base nos códigos SIC de dois dígitos, também presentes nas Enterprise Surveys, para análise das variáveis dicotômicas de cada setor (para controle dos efeitos de setor).

Na Tabela 1 está uma síntese agregada das hipóteses e das variáveis da investigação, dos indicadores utilizados, das transformações realizadas e das fontes.

\section{RESULTADOS}

As variáveis e seus dados descritivos, como forma de fazer transparecer a estrutura dos dados utilizada, são apresentados na Tabela 2.

Para assegurar a inexistência de problemas de altas correlações e de multicolinearidade, estão demonstradas na Tabela 3 as correlações derivadas de nossos testes. Vale informar que testes para diagnóstico de multicolinearidade foram realizados adicionalmente, mas não se encontrou variance inflation factors acima de 1,5, o que atesta a inexistência de problemas

\begin{tabular}{lccccc}
\hline \multicolumn{1}{c}{ Variáveis } & N & Mínimo & Máximo & Média & $\begin{array}{c}\text { Des- } \\
\text { vio-pa- } \\
\text { drão }\end{array}$ \\
\hline Novo produto & 11446 & 0 & 1 &, 38 &, 486 \\
Novo processo & 11446 & 0 & 1 &, 48 &, 500 \\
Investiu em P\&D & 11446 & 0 & 1 &, 62 &, 485 \\
Reformas crescentes & 11446 & 0 & 1 &, 22 &, 415 \\
Reformas decrescentes & 11446 & 0 & 1 &, 09 &, 289 \\
Reversões crescentes & 11446 & 0 & 1 &, 09 &, 287 \\
Reversões decrescentes & 11446 & 0 & 1 &, 04 &, 191 \\
Variação do PIB & 11446 & $-1,49$ & 11,14 & 4,82 & 2,22 \\
PIB per capita & 11446 & 1233,59 & 16245,60 & 6619,67 & 3721,24 \\
Inflação & 11446 & 0,00 & 28,19 & 3,28 & 2,69 \\
Idade da empresa & 11446 & 0 & 210 & 26,44 & 19,877 \\
Posse estrangeira & 11446 & 0 & 100 & 9,19 & 27,282 \\
Posse governamental & 11446 & 0 & 100 &, 12 & 2,698 \\
Número de empregados & 11446 & 1 & 21955 & 134,77 & 526,874 \\
\hline
\end{tabular}

\section{Tab. 02}

Dados descritivos

Fonte: Elaborada pelos autores

inflacionários nas variáveis. As correlações mais elevadas estão apenas entre as variáveis dependentes, indicando que a introdução de novos produtos e de processos está diretamente ligada aos investimentos em P\&D, como já era esperado.
Na Tabela 4, por meio de uma regressão logística, demonstramos os resultados dos testes das hipóteses, tendo a variável dependente, como a introdução de novo produto, assumindo os valores "1" para "sim" e "0" para "não". O Modelo 1 traz apenas o resultado das variáveis de controle. No Modelo 2, testamos a Hipótese 1, acerca da influência positiva das reformas crescentes para a introdução de novos produtos; sendo que, com essa variável dependente, ela não foi confirmada. No Modelo 3, testamos a Hipótese 2, sobre a relação negativa entre reformas decrescentes e a introdução de novos produtos; que foi confirmada, isto é, reformas decrescentes diminuem a introdução de novos produtos. No Modelo 4, testamos a Hipótese 3, que previa uma relação negativa entre reversões crescentes e a introdução de novos produtos. Os resultados, surpreendentemente, mostram o contrário, que quando há reversões crescentes, os pequenos negócios tendem a introduzir novos produtos. No Modelo 5, foi testada a Hipótese 4, em que reversões decrescentes influenciariam positivamente a introdução de novos produtos. Os resultados não confirmaram a hipótese, pois indicaram não haver relação entre as duas variáveis. No Modelo 6, todas as variáveis foram testadas em conjunto, para avaliar, também em conjunto, o tamanho dos seus efeitos. Foram, assim, confirmados os efeitos das reformas decrescentes e das reversões crescentes.

A Tabela 5 contém os testes de regressão logística para a introdução de novos processos, utilizando a variável dicotômica "1" para "sim" e "0" para não. A ordem dos modelos segue a mesma lógica da Tabela 4. 0 Modelo 7 demonstra os efeitos das variáveis de controle; e o Modelo 8, os efeitos das reformas crescentes, testando a Hipótese 1, que não foi confirmada pelos resultados. 0 Modelo 9 demonstra o teste da Hipótese 2, que foi confirmada, pois os resultados indicam um efeito negativo das reformas decrescentes para a introdução de novos processos. 0 Modelo 10 testou a Hipótese 3, que novamente foi contrariada, visto que, diante de reversões crescentes, os pequenos negócios preferem investir em inovação, ao invés de ter estratégias mais conservadoras. No Modelo 11, testamos a Hipótese 4, sendo que o resultado não permitiu verificar efeitos de reversões decrescentes na introdução de novos processos. No Modelo 12, demonstramos o conjunto, confirmando apenas o efeito das reversões decrescentes na introdução de novos processos.

Como forma de analisar os efeitos das dinâmicas institucionais no investimento em $\mathrm{P} \& \mathrm{D}$, realizamos os testes de regressão logística com a variável "a empresa investiu em pesquisa e desenvolvimento no último ano", assumindo os valores "1" para "sim" e "0" para "não".

\begin{tabular}{|c|c|c|c|c|c|c|c|c|c|c|c|c|c|c|}
\hline & & 1 & 2 & 3 & 4 & 5 & 6 & 7 & 8 & 9 & 10 & 11 & 12 & 13 \\
\hline 1 & Novo produto & 1,000 & & & & & & & & & & & & \\
\hline 2 & Novo processo &, $414^{* *}$ & 1,000 & & & & & & & & & & & \\
\hline 3 & Investiu em P\&D &, $309 * *$ &, $333^{* *}$ & 1,000 & & & & & & & & & & \\
\hline 4 & Reformas crescentes &,- 013 &,$- 036^{* *}$ &,$- 094^{* *}$ & 1,000 & & & & & & & & & \\
\hline 5 & Reformas decrescentes &,$- 036^{* *}$ &,- 005 &, $049^{* *}$ &,$- 170^{* *}$ & 1,000 & & & & & & & & \\
\hline 6 & Reversões crescentes &, $090^{* *}$ &, $090 * *$ &, $074^{* *}$ &,$- 168^{* *}$ &,$- 100 * *$ & 1,000 & & & & & & & \\
\hline 8 & Variação do PIB &,$- 045^{* *}$ &,$- 085^{* *}$ &,$- 164^{* *}$ &, $023^{*}$ &, $036^{* *}$ &,$- 262^{* *}$ &, $036^{* *}$ & 1,000 & & & & & \\
\hline 9 & PIB per capita &, $030^{* *}$ &, $050^{* *}$ &,- 006 & ,108** & ,010 &,$- 257^{* *}$ &,$- 208^{* *}$ &, $049 * *$ & 1,000 & & & & \\
\hline 10 & Inflação &,- 007 &,$- 050^{* *}$ &, $029^{* *}$ &,$- 097^{* *}$ &,$- 093^{* *}$ &,$- 100^{* *}$ &, $287^{* *}$ &,$- 115^{* *}$ &,$- 288^{* *}$ & 1,000 & & & \\
\hline 11 & Idade da empresa &,$- 058^{* *}$ &,$- 036^{* *}$ &,$- 105^{* *}$ &,$- 033^{* *}$ &, 010 &,- 018 &,- 006 &, $038^{* *}$ &, $102^{* *}$ &,$- 055^{* *}$ & 1,000 & & \\
\hline 12 & Posse estrangeira &,$- 076^{* *}$ &,$- 047^{* *}$ &,$- 103^{* *}$ &, 014 & ,018 &, $033^{* *}$ &,$- 022^{*}$ &, 010 &,- 003 &,$- 036^{* *}$ &, $027^{* *}$ & 1,000 & \\
\hline 13 & Posse governamental &, 004 &,- 005 &,$- 020^{*}$ &,- 004 &,- 014 &, 001 & ,003 &,- 004 &,$- 025^{* *}$ & ,011 &, $020^{*}$ &, $057^{* *}$ & 1,000 \\
\hline 14 & Número de empregados &,$- 183^{* *}$ &,$- 178^{* *}$ &,$- 301^{* *}$ &, $089 * *$ &, 002 &,$- 036 * *$ &,- 017 &, $091^{* *}$ &, $113^{* *}$ &,$- 080^{* *}$ &, $301^{* *}$ &, $290^{* *}$ &, $047^{* *}$ \\
\hline
\end{tabular}

Tab. 03

Correlações

Fonte: Elaborada pelos autores 


\begin{tabular}{|c|c|c|c|c|c|c|c|c|c|c|c|c|}
\hline \multirow{2}{*}{$\begin{array}{l}\text { Introdução de novo produto } \\
\text { (variável dependente) }\end{array}$} & \multicolumn{2}{|c|}{ Modelo 1} & \multicolumn{2}{|c|}{ Modelo 2} & \multicolumn{2}{|c|}{ Modelo 3} & \multicolumn{2}{|c|}{ Modelo 4} & \multicolumn{2}{|c|}{ Modelo 5} & \multicolumn{2}{|c|}{ Modelo 6} \\
\hline & B & $\mathbf{P}$ & B & $\mathbf{p}$ & B & p & B & p & B & p & B & $\mathbf{p}$ \\
\hline Reformas crescentes & & &,- 018 & ,730 & & & & & & & 068 & ,214 \\
\hline Reformas decrescentes & & & & &,- 289 & 000 & & & & &,- 186 & 015 \\
\hline Reversões crescentes & & & & & & & 641 & 000 & & & 634 & 000 \\
\hline Reversões decrescentes & & & & & & & & &,- 070 & ,523 & 015 & 891 \\
\hline Variação do PIB &,- 061 & 000 &,- 061 & 000 &,- 055 & 000 &,- 038 & 001 &,- 061 & 000 &,- 034 & 003 \\
\hline PIB per capita &, 000 & 000 & 000 & ,000 &, 000 & 000 &, 000 & 000 &, 000 & 000 &, 000 & 000 \\
\hline Inflação &,- 003 & ,743 &,- 003 & ,738 &,- 006 & ,434 & 006 & ,456 &,- 002 & 830 & 004 & 665 \\
\hline Idade da empresa &,- 005 & 000 &,- 005 & 000 &,- 005 & 000 &,- 005 & 000 &,- 005 & 000 &,- 005 & 000 \\
\hline Posse estrangeira &,- 004 & 000 &,- 004 & 000 &,- 004 & 000 &,- 004 & 000 &,- 004 & 000 &,- 004 & 000 \\
\hline Posse governamental & ,013 & 083 & 013 & 084 & ,013 & 092 & ,014 & 063 & 013 & 084 &, 014 & 067 \\
\hline Número de empregados &,- 001 & 000 &,- 001 & 000 &,- 001 & 000 &,- 001 & 000 &,- 001 & 000 &,- 001 & 000 \\
\hline Controles de setor (dummy) & Sim & & Sim & & Sim & & Sim & & Sim & & Sim & \\
\hline Verossimilhança de log -2 & 14828 & & 14828 & & 14813 & & 14752 & & 14828 & & 14744 & \\
\hline R quadrado (Nagelkerke) & 0,05 & &, 047 & &, 049 & &, 056 & &, 047 & &, 057 & \\
\hline Reg. Sig & $\mathrm{p}<0,000$ & & $\mathrm{p}<0,000$ & & $\mathrm{p}<0,000$ & & $\mathrm{p}<0,000$ & & $\mathrm{p}<0,000$ & & $\mathrm{p}<0,000$ & \\
\hline
\end{tabular}

Tab. 04

Testes para a introdução de novos produtos

Fonte: Elaborada pelos autores

Na Tabela 6, testamos as variáveis de controle no Modelo 13. No Modelo 14, testamos a Hipótese 1, sendo que o resultado apresentou um efeito contrário ao previsto. No Modelo 15, testamos a Hipótese 2, que foi contrariada no resultado, indicando um efeito oposto. Esse efeito também foi observado na Hipótese 3, testada no Modelo 16. 0 Modelo 17 testou a Hipótese 4, não a confirmando. Os efeitos se mantêm no Modelo 18 , que testa todas as variáveis em conjunto.

\section{DISCUSSÕES}

Este estudo teve como propósito analisar os efeitos das dinâmicas institucionais nos esforços de inovação dos pequenos negócios. Para isso, realizamos uma análise com 11.446 pequenos negócios de países latino-americanos, classificando as dinâmicas institucionais como reformas institucionais prómercado, crescentes e decrescentes, e reversões institucionais crescentes e decrescentes. Testamos os efeitos dessas dinâmicas em três variáveis dependentes, que representam os esforços de inovação dos pequenos negócios. Ao contrário do que esperávamos, os resultados são diferentes, conforme o tipo de esforços de inovação realizados.
As reformas crescentes, propostas como possíveis fomentadoras dos esforços de inovação nos pequenos negócios (Dau, 2012; Barasa et al., 2017), não tiveram efeito, quando analisadas em relação à introdução de novos produtos e de novos processos. Já no que se refere aos investimentos em P\&D, quando há reformas crescentes, menos investimentos são percebidos. Esse efeito contrário ao esperado faz sentido, se pensarmos que tais investimentos não melhoram apenas a posição dos pequenos negócios latino-americanos, garantindo, igualmente, sua sobrevivência, face a um possível aumento nas dificuldades. Sendo assim, quando a perspectiva é de melhora institucional (Cuervo-Cazurra and Dau, 2009; Banalieva et al., 2018), os pequenos negócios param de investir em P\&D e focam seus recursos em aproveitar o momento positivo no mercado.

No caso da presença de reformas institucionais prómercado decrescentes, foi proposto que elas teriam um efeito negativo nos esforços de inovação dos pequenos negócios (Dau and Cuervo-Cazurra, 2014; Barasa et al., 2017). Os resultados demonstram que os pequenos negócios realmente têm menos esforços de inovação, em relação à introdução de novos produtos e de processos, quando encaram reformas decrescentes, pois tendem a buscar estratégias mais conservadoras, em condições de incerteza. Os investimentos em P\&D, por sua vez, aumentam

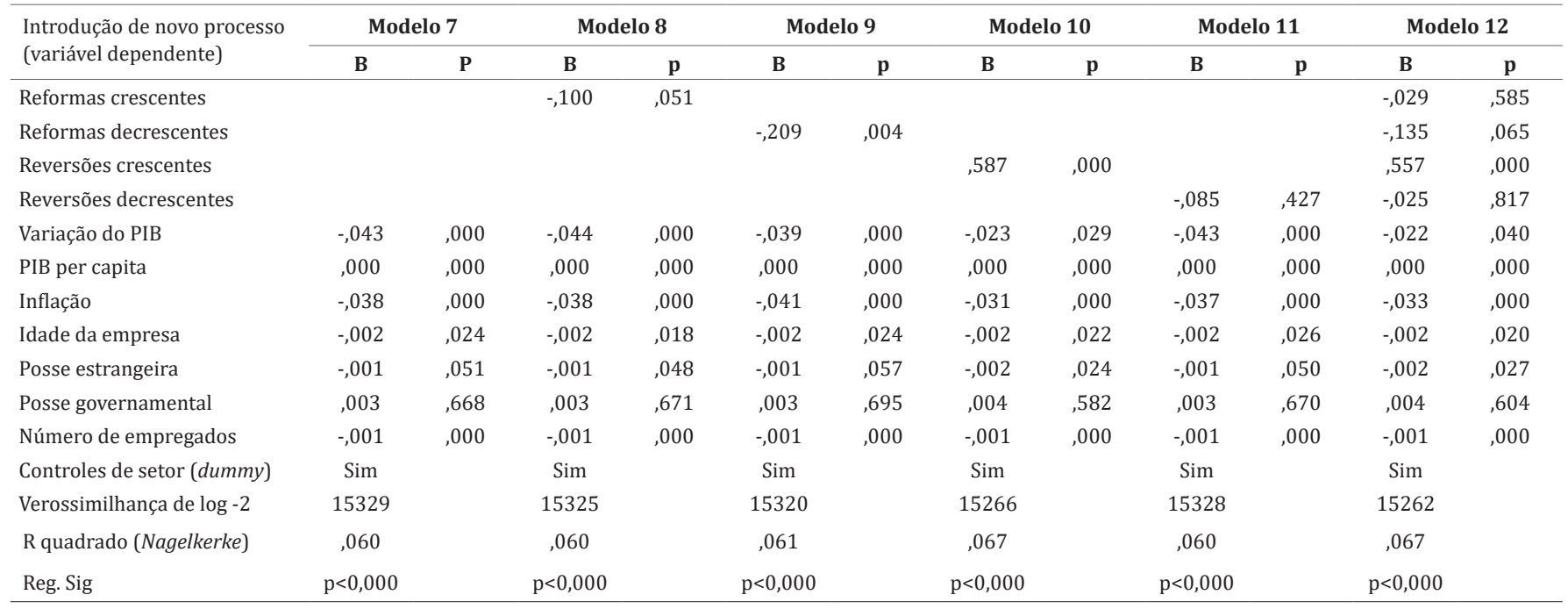

Tab. 05

Testes para a introdução de novos processos

Fonte: Elaborada pelos autores 


\begin{tabular}{|c|c|c|c|c|c|c|c|c|c|c|c|c|}
\hline \multirow{2}{*}{$\begin{array}{l}\text { Investimento em P\&D } \\
\text { (variável dependente) }\end{array}$} & \multicolumn{2}{|c|}{ Modelo 13} & \multicolumn{2}{|c|}{ Modelo 14} & \multicolumn{2}{|c|}{ Modelo 15} & \multicolumn{2}{|c|}{ Modelo 16} & \multicolumn{2}{|c|}{ Modelo 17} & \multicolumn{2}{|c|}{ Modelo 18} \\
\hline & B & $\mathbf{P}$ & B & $\mathbf{p}$ & B & p & B & p & B & $\mathbf{p}$ & B & $\mathbf{p}$ \\
\hline Reformas crescentes & & &,- 294 & ,000 & & & & & & &,- 243 & 000 \\
\hline Reformas decrescentes & & & & & 176 & ,027 & & & & & 194 & 017 \\
\hline Reversões crescentes & & & & & & & 333 &, 000 & & & 290 & 001 \\
\hline Reversões decrescentes & & & & & & & & & 065 &, 549 & ,073 & ,509 \\
\hline Variação do PIB &,- 120 & ,000 &,- 125 & 000 &,- 123 &, 000 &,- 109 & 000 &,- 120 & 000 &,- 119 & 000 \\
\hline PIB per capita &, 000 & 000 &, 000 & 000 & ,000 &, 000 &, 000 &, 000 &, 000 & 000 &, 000 & 000 \\
\hline Inflação &,- 021 & 017 &,- 023 & 009 &,- 019 & 028 &,- 016 & ,076 &,- 022 & 014 &,- 018 & 053 \\
\hline Idade da empresa &,- 006 & ,000 &,- 006 & ,000 &,- 006 &, 000 &,- 006 &, 000 &,- 006 & ,000 &,- 006 & 000 \\
\hline Posse estrangeira &,- 003 & ,000 &,- 003 & ,000 &,- 003 & ,000 &,- 003 & ,000 &,- 003 & ,000 &,- 003 & 000 \\
\hline Posse governamental &, 002 & 844 &, 001 & ,864 & ,002 & ,821 &, 002 & 799 &, 002 & 842 &, 002 & ,793 \\
\hline Número de empregados &,- 001 & ,000 &,- 001 & ,000 &,- 001 &, 000 &,- 001 & ,000 &,- 001 & ,000 &,- 001 & 000 \\
\hline Controles de setor (dummy) & Sim & & $\operatorname{Sim}$ & & Sim & & Sim & & Sim & & Sim & \\
\hline Verossimilhança de log -2 & 13907 & & 13877 & & 13902 & & 13890 & & 13906 & & 13862 & \\
\hline R quadrado (Nagelkerke) & ,143 & & ,146 & &, 143 & &, 144 & & ,143 & & ,147 & \\
\hline Reg. Sig & $\mathrm{p}<0,000$ & & $\mathrm{p}<0,000$ & & $\mathrm{p}<0,000$ & & $\mathrm{p}<0,000$ & & $\mathrm{p}<0,000$ & & $\mathrm{p}<0,000$ & \\
\hline
\end{tabular}

Tab. 06

Testes para o investimento em P\&D

Fonte: Elaborada pelos autores

na presença de reformas decrescentes, efeito que pode estar relacionado a um foco maior no longo prazo. Nesse sentido, os pequenos negócios, ao perceberem reformas decrescentes (Cuervo-Cazurra and Dau, 2009; Banalieva et al., 2018), passam a investir em P\&D para garantir uma vantagem competitiva em relação aos seus concorrentes, no caso de uma possível deterioração institucional no futuro.

Sobre as reversões institucionais crescentes, o resultado apontou efeitos contrários aos previstos como negativos, isto é, ao invés de uma deterioração institucional cada vez maior diminuir os esforços de inovação dos pequenos negócios (Dau and Cuervo-Cazurra, 2014; Barasa et al., 2017), ela aumentou a incidência de esforços de inovação (introdução de novos produtos e de processos, e investimentos em P\&D). Os resultados podem indicar, ao contrário do que supomos, que os pequenos negócios, diante de grande deterioração institucional e de incertezas futuras (Dau and Cuervo-Cazurra, 2014; Banalieva et al., 2018), preferem realizar esforços de inovação, talvez como forma de garantir vantagem ou paridade competitiva, e de sobreviver. Por outro lado, não houve relação alguma entre as reversões decrescentes e os esforços de inovação. A síntese agregada das implicações do teste de hipótese está apresentada na Tabela 7.

\section{CONCLUSÕES}

Com base nos resultados, concluímos que, nos pequenos negócios de países latino-americanos, os esforços de inovação podem estar mais ligados à preparação para enfrentar um ambiente institucionalmente ineficiente do que para aproveitar aqueles que passaram por uma melhora institucional. Esse movimento pode ser interpretado como a busca por sobrevivência em um ambiente incerto, contrariando os efeitos esperados, de que haveria mais esforços de inovação quando o ambiente fosse propício a ela.

Este estudo avaliou conjunta e empiricamente fatores econômicos de dinâmicas institucionais (reformas e reversões), explicando os esforços de inovação dos pequenos negócios (alocação de recursos), em um número alargado de países latino-americanos; possibilitou a análise dos tipos de dinâmicas institucionais e de suas respectivas respostas, na forma de esforços de inovação; e demonstrou como a formulação de reformas institucionais pode impactar os pequenos negócios de países latino-americanos, contribuindo, desse modo, para a ampliação da literatura sobre o tema e para o desenvolvimento de políticas públicas.

A pesquisa, apesar das inúmeras contribuições citadas, teve algumas limitações, como o fato de ela ter sido realizada com

\begin{tabular}{|c|c|c|}
\hline \multicolumn{2}{|c|}{ Reformas e reversões institucionais: fundamentação teórica } & \multirow{2}{*}{$\begin{array}{l}\text { Implicações do teste de hipótese } \\
\text { (H1) Quando há reformas institucionais crescentes, há } \\
\text { menos investimento em P\&D. No entanto, essas reformas } \\
\text { não tiveram efeito, quando analisadas em relação à intro- } \\
\text { dução de novos produtos e de novos processos. }\end{array}$} \\
\hline $\begin{array}{l}\text { Reformas institucionais são mudanças insti- } \\
\text { tucionais pró-mercado e tendem a diminuir } \\
\text { a intervenção do governo nos negócios e na } \\
\text { economia, resultando em maior liberdade e }\end{array}$ & $\begin{array}{l}\text { Reformas institucionais crescentes: quando uma melhora } \\
\text { institucional é seguida por uma melhora ainda maior, no } \\
\text { ano seguinte (Banalieva et al., 2018). }\end{array}$ & \\
\hline $\begin{array}{l}\text { oportunidade para as empresas em um pais } \\
\text { (Cuervo-Cazurra and Dau, 2009). }\end{array}$ & $\begin{array}{l}\text { Reformas institucionais decrescentes: quando uma mel- } \\
\text { hora institucional é seguida por uma melhora relativa- } \\
\text { mente menor à primeira, no ano subsequente (Banalieva } \\
\text { et al., 2018). }\end{array}$ & $\begin{array}{l}\text { (H2) Quando há reformas institucionais decrescentes, há } \\
\text { menos esforços de introdução de novos produtos e proces- } \\
\text { sos. Entretanto, os investimentos em P\&D aumentam na } \\
\text { presença dessas reformas. }\end{array}$ \\
\hline \multirow{2}{*}{$\begin{array}{l}\text { Reversões institucionais são retrocessos insti- } \\
\text { tucionais e tendem a aumentar a intervenção } \\
\text { do governo nos negócios e na economia, } \\
\text { resultando em menor liberdade e oportuni- } \\
\text { dade para as empresas em um país (Dau and } \\
\text { Cuervo-Cazurra, 2014). }\end{array}$} & $\begin{array}{l}\text { Reversões institucionais crescentes: quando uma dete- } \\
\text { rioração institucional é seguida por uma deterioração } \\
\text { ainda maior, no ano subsequente (Banalieva et al., 2018). }\end{array}$ & $\begin{array}{l}\text { (H3) Quando há reversões institucionais crescentes, há } \\
\text { mais esforços de introdução de novos produtos e proces- } \\
\text { sos. }\end{array}$ \\
\hline & $\begin{array}{l}\text { Reversões institucionais decrescentes: quando uma de- } \\
\text { terioração institucional é seguida por uma deterioração } \\
\text { menor, no ano subsequente (Banalieva et al., 2018). }\end{array}$ & $\begin{array}{l}\text { (H4) Reformas institucionais crescentes não tiveram efeito, } \\
\text { quando analisadas em relação aos esforços de inovação } \\
\text { (introdução de novos produtos e processos, e investimento } \\
\text { em P\&D). }\end{array}$ \\
\hline
\end{tabular}

Tab. 07

Síntese agregada das implicações do teste de hipótese

Fonte: Elaborada pelos autores 
empresas de todos os tipos etamanhos (coma predominância dos pequenos negócios, que representam $74 \%$ da amostra), sendo que, muitas delas não puderam precisar o grau de inovação dos esforços realizados. Isso torna a medida de inovação utilizada passível de críticas, talvez por representar um esforço incipiente de inovação. No entanto, ressalta-se que pequenas e médias empresas constituem uma parcela importante na economia dos países latino-americanos, que não pode ser desconsiderada. Sendo assim, apesar de simples, essa medida é a única possível para esse tipo de negócio.

Outra limitação está na amostra considerada: a consecução da Enterprise Survey demanda uma quantidade muito grande de recursos, não sendo possível, portanto, sua realização anual. Assim, os resultados alcançados têm por base uma amostra de países com apenas dois ou três anos focais. Estudos futuros poderiam sanar essas limitações e complementar nossos resultados, analisando dados secundários, disponibilizados todos os anos, como as patentes submetidas por país, anualmente.

\section{Conflito de Interesses}

Os autores declaram não haver conflitos de interesse.

\section{Agradecimento}

Este estudo recebeu financiamento do projeto de bolsa de pesquisa "672/2019 Qualidade Institucional e as Estratégias Internacionais das Organizações - Universidade Regional de Blumenau"

CNPq UNIVERSAL (Processo 422922/2018-8): Investigação Multimétodo do Efeito do Desempenho Inovador na Performance Econômico-Financeira das Empresas Latino-americanas: Uma Relação Moderada por Gatilhos Relacionais e Fatores Institucionais

CNPq PQ2 (Processo 309294/2019-3): Dinâmicas Relacionais e Institucionais e os Esforços de Inovação na América Latina.

\section{Contribuições Individuais}

\begin{tabular}{rcc}
\multicolumn{1}{c}{ Papéis } & \multicolumn{2}{c}{ Contribuição por autor } \\
\cline { 2 - 3 } & Falaster CD & Costa PR \\
\hline Conceitualização & $\mathrm{X}$ & $\mathrm{X}$ \\
\hline Metodologia & $\mathrm{X}$ & $\mathrm{X}$ \\
\hline Software & $\mathrm{X}$ & $\mathrm{X}$ \\
\hline Validação & $\mathrm{X}$ & $\mathrm{X}$ \\
\hline Análise formal & $\mathrm{X}$ & $\mathrm{X}$ \\
\hline Pesquisa / Levantamento & $\mathrm{X}$ & $\mathrm{X}$ \\
\hline Recursos & $\mathrm{X}$ & $\mathrm{X}$ \\
\hline Curadoria dos dados & $\mathrm{X}$ & $\mathrm{X}$ \\
\hline Escrita - Rascunho original & $\mathrm{X}$ & $\mathrm{X}$ \\
\hline Escrita - Revisão e edição & $\mathrm{X}$ & $\mathrm{X}$ \\
\hline Visualização dos dados & $\mathrm{X}$ & $\mathrm{X}$ \\
\hline Supervisão / Orientação & $\mathrm{X}$ & $\mathrm{X}$ \\
\hline Administração do Projeto & $\mathrm{X}$ & $\mathrm{X}$ \\
\hline Financiamento & $\mathrm{X}$ & $\mathrm{X}$ \\
\hline
\end{tabular}

\section{REFERÊNCIAS}

Abed, M. G. T., \& Gupta, M. S. (2002). Governance, corruption, and economic performance. Washington, DC: International Monetary Fund.

Acemoglu, D., \& Robinson, J. A. (2008). Persistence of power, elites, and institutions. American Economic Review, 98(1), 267-93. https://doi.org/10.1257/ aer.98.1.267

Alence, R. (2004). Political institutions and developmental governance in subSaharan Africa. The Journal of Modern African Studies, 42(2), 163-187. https://doi.org/10.1080/12265080701694512

Alonso, J. A., \& Garcimartín, C. (2013). The determinants of institutional quality. More on the debate. Journal of International Development, 25(2), 206-226. https://doi.org/10.1002/jid.1710

Arundel, A., Bordoy, C., \& Kanerva, M. (2007). Neglected innovators: How do innovative firms that do not perform R\&D innovate. Results of an analysis of the Innobarometer, 9.

Ayyagari, M., Demirgüç-Kunt, A., \& Maksimovic, V. (2011). Firm innovation in emerging markets: the role of finance, governance, and competition. Journal of Financial and Quantitative Analysis, 46(6), 1545-1580. https://doi. org/10.1017/S0022109011000378

Banalieva, E. R., Cuervo-Cazurra, A., \& Sarahi, R. (2018). Dynamics of pro-market institutions and firm performance. Journal of International Business Studies, 49(7), 858-880. https://doi.org/10.1057/s41267-018-0155-7

Barasa, L., Knoben, J., Vermeulen, P., Kimuyu, P., \& Kinyanjui, B. (2017). Institutions, resources and innovation in East Africa: A firm level approach. Research Policy, 46(1), 280-291. https://doi.org/10.1016/j.respol.2016.11.008

Barney, J., \& Arikan, A.M. (2001). The resource-based view: origins and implications. In Hitt, M. A., Freeman, R. E., Harrison, J. S. (Eds.), Handbook of Strategic Management (pp. 124-188). Oxford: Blackwell Publishers.

Bhattacharya, U., Hsu, P. H., Tian, X., \& Xu, Y. (2017). What affects innovation more: Policy or policy uncertainty? Journal of Financial and Quantitative Analysis, 52(5), 1869-1901. https://doi.org/10.1017/S0022109017000540

Bradley, S. W., McMullen, J. S., Artz, K., \& Simiyu, E. M. (2012). Capital is not enough: Innovation in developing economies. Journal of Management Studies, 49(4), 684-717. https://doi.org/10.1111/j.1467-6486.2012.01043.x

Bräutigam, D. A., \& Knack, S. (2004). Foreign aid, institutions, and governance in sub-Saharan Africa. Economic development and cultural change, 52(2), 255285.

Crespi, G., \& Zuniga, P. (2012). Innovation and productivity: evidence from six Latin American countries. World development, 40(2), 273-290. https://doi. org/10.1016/j.worlddev.2011.07.010

Cuervo-Cazurra, A., \& Dau, L. A. (2009). Structural reform and firm exports. Management International Review, 49(4), 479-507. https://doi.org/10.1007/ s11575-009-0005-8

Dau, L. A. (2012). Pro-market reforms and developing country multinational corporations. Global Strategy Journal, 2(3), 262-276. https://doi.org/ 10.1111/j.2042-5805.2012.01033.x

Dau, L. A. (2013). Learning across geographic space: Pro-market reforms, multinationalization strategy, and profitability. Journal of International Business Studies, 44(3), 235-262. https://doi.org/10.1057/jibs.2013.5

Dau, L. A., \& Cuervo-Cazurra, A. (2014). To formalize or not to formalize: Entrepreneurship and pro-market institutions. Journal of Business Venturing, 29(5), 668-686. https://doi.org/10.1016/i.jbusvent.2014.05.002

Freel, M. S. (2005). Patterns of innovation and skills in small firms. Technovation, 25(2), 123-134.

Glaeser, E. L., La Porta, R., Lopez-de-Silanes, F., \& Shleifer, A. (2004). Do institutions cause growth?. Journal of economic Growth, 9(3), 271-303. https://doi. org/10.1023/B:JOEG.0000038933.16398.ed

Goedhuys, M. (2007). Learning, product innovation, and firm heterogeneity in developing countries; Evidence from Tanzania. Industrial and Corporate Change, 16(2), 269-292. https://doi.org/10.1093/icc/dtm003

Goedhuys, M., Janz, N., \& Mohnen, P. (2013). Knowledge-based productivity in "low-tech" industries: evidence from firms in developing countries. Industrial and Corporate Change, 23(1), 1-23. https://doi.org/10.1093/icc/dtt006

Goedhuys, M., \& Sleuwaegen, L. (2010). High-growth entrepreneurial firms in Africa: a quantile regression approach. Small Business Economics, 34(1), 3151. https://doi.org/10.1007/s11187-009-9193-7

Goedhuys, M., \& Veugelers, R. (2012). Innovation strategies, process and product innovations and growth: Firm-level evidence from Brazil. Structural change and economic dynamics, 23(4), 516-529. https://doi.org/10.1016/j strueco.2011.01.004

Greif, A. (2006). Family structure, institutions, and growth: the origins and implications of western corporations. American economic review, 96(2), 308312. https://doi.org/10.1257/000282806777212602

Hair, J., Babin, B., Money, A., \& Samouel, P. (2005). Fundamentos de métodos de pesquisa em administração. Bookman Companhia Ed.

Henisz, W. J. (2000). The institutional environment for economic growth. Economics \& Politics, 12(1), 1-31. https://doi.org/10.1111/1468-0343.00066

Ireland, R. D., Hitt, M. A., \& Sirmon, D. G. (2003). A model of strategic entrepreneurship: The construct and its dimensions. Journal of management, 29(6), 963-989. https://doi.org/10.1016/S0149-2063(03)00086-2 
Kafouros, M., Wang, C., Piperopoulos, P., \& Zhang, M. (2015). Academic collaborations and firm innovation performance in China: The role of region-specific institutions. Research Policy, 44(3), 803-817. https://doi. org/10.1016/j.respol.2014.11.002

Kamau, P., \& Munandi, I. (2009). Innovation in the Kenyan Clothing Sector and Its Impact on Employment and Poverty Reduction. Institute for Development Studies, University of Nairobi, Nairobi, Kenya.

Kannebley Jr., S., Porto, G. S., \& Pazello, E. T. (2005). Characteristics of Brazilian innovative firms: An empirical analysis based on PINTEC - industrial research on technological innovation. Research Policy, 34(6), 872-893. https://doi. org/10.1016/j.respol.2005.04.003

Kaufmann, D., Kraay, A., \& Mastruzzi, M. (2011). The worldwide governance indicators: methodology and analytical issues. Hague Journal on the Rule of Law, 3(2), 220-246. https://doi.org/10.1017/S1876404511200046

Lee, K., \& Kang, S. M. (2007). Innovation types and productivity growth: Evidence from Korean manufacturing firms. Global Economic Review, 36(4), 343-359. https://doi.org/10.1080/12265080701694512

Mahoney, J. T. (1995). The management of resources and the resource of management. Journal of business research, 33(2), 91-101. https://doi. org/10.1016/0148-2963(94)00060-R

Nelson, R. R., \& Nelson, K. (2002). Technology, institutions, and innovation systems. Research policy, 31(2), 265-272. https://doi.org/10.1016/S00487333(01)00140-8

North, D. C. (1990). Institutions, Institutional Change and Economic Performance. Cambridge, UK: Cambridge University Press.

North, D. C. (1991). Institutions. Journal of economic perspectives, 5(1), 97-112.

OECD. (2005). Oslo manual: Guidelines for collecting and interpreting innovation data (3rd ed). Paris: OCDE.

Olson, M., Sarna, N., \& Swamy, A. V. (2000). Governance and growth: A simple hypothesis explaining cross-country differences in productivity growth. Public Choice, 102(3-4), 341-364. https://doi.org/10.1023/A:1005067115159

Papazoglou, M. E., \& Spanos, Y. E. (2018). Bridging distant technological domains: A longitudinal study of the determinants of breadth of innovation diffusion. Research Policy, 47(9), 1713-1728. https://doi.org/10.1016/j. respol.2018.06.006

Rosenberg, N. (2010). Why do firms do basic research (with their own money)? In Studies On Science And The Innovation Process (pp. 225-234). Stanford University, USA: World Scientific. https://doi. org/10.1142/9789814273596_0011

Sirmon, D. G., Hitt, M. A., \& Ireland, R. D. (2007). Managing firm resources in dynamic environments to create value: Looking inside the black box. Academy of management review, 32(1), 273-292. https://doi.org/10.5465/ amr.2007.23466005

Solleiro, J. L., \& Castañón, R. (2005). Competitiveness and innovation systems: the challenges for Mexico's insertion in the global context. Technovation, 25(9), 1059-1070. https://doi.org/10.1016/j.technovation.2004.02.005
Szogs, A. (2008). The role of mediator organisations in the making of innovation systems in least developed countries: evidence from Tanzania. International Journal of Technology and Globalisation, 4(3), 223. https://doi.org/10.1504/ IJTG.2008.020328

Tolstoy, D. (2009). Knowledge combination and knowledge creation in a foreignmarket network. Journal of Small Business Management, 47(2), 202-220.

Trevino, L. J., Thomas, D. E., \& Cullen, J. (2008). The three pillars of institutional theory and FDI in Latin America: An institutionalization process International Business Review, 17(1), 118-133. https://doi.org/10.1016/i. ibusrev.2007.10.002

Wang, J. (2018). Innovation and government intervention: A comparison of Singapore and Hong Kong. Research Policy, 47(2), 399-412. https://doi. org/10.1016/j.respol.2017.12.008

Wang, C. C., \& Lin, G. C. (2012). Dynamics of innovation in a globalizing china: regional environment, inter-firm relations and firm attributes. Journal of Economic Geography, 13(3), 397-418. https://doi.org/10.1093/jeg/lbs019

Williamson, 0. E. (2000). The new institutional economics: taking stock, looking ahead. Journal of economic literature, 38(3), 595-613. https://doi. org/10.1257/jel.38.3.595

World Bank. (2019). Enterprise Surveys. Recuperado de http://www. enterprisesurveys.org/data

Wu, J., \& Park, S. H. (2019). The role of international institutional complexity on emerging market multinational companies' innovation. Global Strategy Journal, 9(2), 333-353. https://doi.org/10.1002/gsj.1166

\section{BIOGRAFIA DOS AUTORES}

Christian Daniel Falaster é professor de Estratégia e Negócios Internacionais na Universidade Regional de Blumenau (FURB). Blumenau, Santa Catarina - Brasil. Recebeu seu Doutorado pela Universidade Nove de Julho. Tem interesse nas áreas de fusões e aquisições, instituições e desempenho.

Priscila Rezende da Costa é professora e diretora do programa de pós-graduação em administração da Universidade Nove de Julho (UNINOVE), São Paulo, SP, Brasil. Recebeu seu Doutorado pela Universidade de São Paulo (FEA/USP), São Paulo, SP, Brasil. Tem interesse nas áreas de Inovação, Cooperação entre empresas e universidades, Capacidades dinâmicas, Capacidade relacional, Capacidade absortiva, e Internacionalização da Inovação. 